\title{
L'ingénieur Krebs (1850-1935) ou comment traduire le fluide électrique
}

\section{Philippe Krebs}

\section{(2) OpenEdition}

1 Journals

\section{Édition électronique}

URL : https://journals.openedition.org/artefact/7663

DOI : 10.4000/artefact.7663

ISSN : 2606-9245

Éditeur:

Association Artefact. Techniques histoire et sciences humaines, Presses universitaires du Midi

\section{Édition imprimée}

Date de publication : 18 juin 2015

Pagination : $27-47$

ISBN : 978-2-271-08155-1

ISSN : 2273-0753

\section{Référence électronique}

Philippe Krebs, «L'ingénieur Krebs (1850-1935) ou comment traduire le fluide électrique », Artefact [En ligne], HS 01 | 2015, mis en ligne le 30 avril 2021, consulté le 05 mai 2021. URL : http:// journals.openedition.org/artefact/7663; DOI : https://doi.org/10.4000/artefact.7663

\section{c) $(1)$}

Artefact, Techniques, histoire et sciences humaines est mise à disposition selon les termes de la Licence Creative Commons Attribution - Pas d'Utilisation Commerciale - Pas de Modification 4.0 International. 


\section{L'ingénieur Krebs (1850-1935) ou comment traduire le fluide électrique}

Philippe KREBS*

\section{Résumé}

Les ingénieurs du XIX ${ }^{\mathrm{e}}$ siècle ont largement manié le modèle épistémologique du fluide pour répondre aux attentes des milieux socio-économiques de leur temps. Dans le domaine de la locomotion et de la motricité, la vapeur est l'énergie de la puissance, pesante et dangereuse, et l'électricité est la nouvelle énergie universelle promise à tous les succès. L'ingénieur militaire Arthur Krebs, à la recherche de la maîtrise de la matière et de l'énergie, conçoit des objets électriques novateurs, de mobilité aérostatique et sous-marine comme des ensembles continus de fluides collectés et transportés. La jeune république française en reconstruction a des rêves de modernité et d'autonomie. L'ingénieur réorganisateur du service d'incendie de la Ville de Paris ne doit plus seulement maîtriser les fluides matériels, il doit également maîtriser le fluide-temps, aussi invisible que le fluide électrique. La vitesse mécanique et électrique est sa réponse aux exigences de fluidité. La pensée de la mobilité, de la vitesse et de l'autonomie le conduisent finalement à se faire le promoteur, chez Panhard-Levassor, de la cause automobile et du moteur à pétrole en apportant des solutions techniques dans la voie de l'hybridation du pétrole et de l'électricité, au point qu'on a pu dire alors que cette dernière avait «sauvé» le moteur à essence face aux moteurs à vapeur et électrique.

Mots-clés : automobile, fluide-temps, hybridation, mobilité, motricité.

\footnotetext{
* Philippe Krebs est consultant en systèmes d'information au sein d'une société éditrice de progiciels de gestion bancaire. De formation en droit des affaires, il obtient un DEA de sociologie du droit et des relations sociales du travail avec Marcel David à l'Institut des sciences sociales du travail de Bourg-la-Reine (ISST-Paris I en 1984), puis consacre son mémoire de master II en histoire des techniques à son aïeul, Arthur Constantin Krebs (Philippe Krebs, Arthur Constantin Krebs (1850-1935). Autorité et stratégie à la direction de Panhard et Levassor (1913-1916), mémoire de master II, sous la dir. de Liliane Hilaire-Pérez, CDHTE-Cnam, Paris, 2009). Il est actuellement doctorant au Centre Alexandre Koyré sous la direction de Liliane Hilaire-Pérez. Contact: philippe.krebs@soprabanking.com.
} 


\section{Abstract}

The engineers of the nineteenth century made wide use of the epistemological model of the fluid in the course of their answers to the expectations of socio-economic milieux of their time. In the field of locomotion and motor, steam was the energy of power, heavy and dangerous, and electricity was the new universal energy promised to success. The military engineer Arthur Krebs, looking for the control of matter and energy, has designed innovative electrical objects of aerostatic and underwater mobility considered as continuous sets of collected fluids, transported. The young Republic of France in reconstruction had dreams of modernity and autonomy. The engineer reorganizing the Fire Department of the City of Paris, no longer only had to control the material fluids; he also had to control the time-fluid, as invisible as the electric fluid was. The mechanical and electrical speed was his answer to fluidity requirements. The thought of mobility, speed and autonomy ultimately lead Arthur Krebs to promote, while working at Panhard-Levassor's, the automotive and oil choices by providing technical solutions in the way of hybridization oil and electricity, so that people said that the electricity had "saved" the gasoline engine in front of the steam and electric engines.

Keywords : automotive, hybridization, mobility, time-fluid, traction.

Durant tout le $\mathrm{XIX}^{\mathrm{e}}$ siècle des sciences et des techniques, les discours portant sur les phénomènes dynamiques sont largement dominés par l'usage de la métaphore du fluide. Archétypique, Sadi Carnot énonce, en 1824, ses principes de la «thermo-mécanique» en associant chaleur et mouvement. Pour décrire les conditions qui permettent de transformer la chaleur en mouvement, il utilise la vieille notion $\mathrm{du}$ «calorique»:

«Peut-on concevoir les phénomènes de la chaleur et de l'électricité comme dus à autre chose qu'à des mouvements quelconques de corps, et comme tels ne doivent-ils pas être soumis aux lois générales de la mécanique ${ }^{1}$ ?»

Avec Jean Mathiot ${ }^{2}$, nous remarquerons qu'ici la démarche de Carnot n'est pas analogique: "le fluide calorique supposé se déplacer de la source chaude vers la source froide est le moyen d'atteindre une formulation analytique qui subsistera lorsque Clausius même aura déraciné [en 1863] la notion de calorique substance.» Chez Carnot, le modèle du fluide, indépendamment de toute thèse réaliste, a une fonction opératoire évidente. Ce rapprochement métaphorique s'avère pertinent, car l'articulation entre la substance et son modèle est porteuse de propriétés heuristiques: par sa capacité à déplacer les significations, elle fait naître des analogies. De ce point de vue et dans ce contexte technologique, la question se pose alors des rapports entre le fluide-matière et le modèle du fluide.

Dans le cadre de son anthropologie des techniques qui analysait les relations de l'homme à la matière, André LeroiGourhan $^{1}$ avait proposé de définir les fluides comme des masses mobiles qu'il faut «emprisonner» pour les maintenir 
en place. Telles sont, par exemple, une quantité d'eau ou une quantité de grains de blé ou de pommes. Il ajoute: "Toute l'étude technique des fluides tiendra donc dans l'étude des objets par lesquels on peut emprisonner, transporter et libérer ces corps.» Ainsi, le maniement de la matière fluide impose-t-il nécessairement la maîtrise de trois modes opératoires: la collection, le transport et le transversement. Les objets répondant à cette définition sont très hétérogènes. $\mathrm{Si}$ la jarre du potier, avec son goulot et sa panse, rassemble en elle-même ces trois modes, la rigole, en revanche, les distingue explicitement. Alimentée à l'une de ses extrémités, sa forme et son inclinaison transportent son contenu jusqu'à son autre extrémité, où celui-ci pourra se déverser. Dans ce dernier cas, le fluide est en circulation et prendra le nom de «flux».

Nous avons vu que, dans la pensée de Sadi Carnot, la chaleur et l'électricité étaient toutes deux représentées comme des fluides. C'est pour expliquer la dynamique des fluides en mouvement qu'il recourt à la célèbre analogie: "on peut comparer avec assez de justesse la puissance motrice de la chaleur à celle d'une chute d'eau ${ }^{4}$.» Ainsi, l'idée du fluide part-elle d'un présupposé, analogue au calorique de Carnot, qu'il s'agit de démontrer expérimentalement: indestructible, le fluide peut subir le mouvement sans être lui-même mouvement. Le monde présupposé par le modèle $\mathrm{du}$ fluide est un monde «homogène, continûment intelligible, sans supposition de nature essentialiste ${ }^{5}$. L'essence $\mathrm{du}$ fluide serait donc phénoménologique, il délimite un contexte, un cadre de connaissance des possibles.

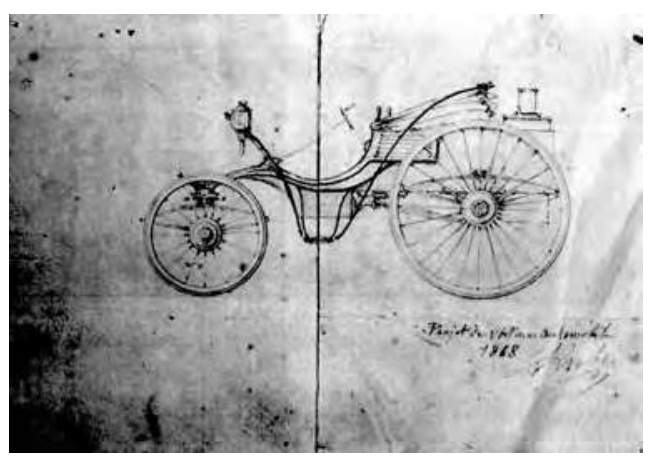

Fig.1. Avec ce "projet d'automobile", probablement exécuté en classe de Math-sup à Besançon, le jeune Arthur Krebs, romantique de 18 ans aux cheveux longs, exprime dès 1868 son rêve de vitesses mécaniques.

Nous tenterons de montrer ici comment l'ingénieur du XIX ${ }^{e}$ siècle manie ce concept de fluide en s'appuyant sur la variation des définitions qui lui sont attachées et les réponses techniques qu'elles suscitent. Le parcours de l'ingénieur Arthur Constantin Krebs, que l'on se proposera de suivre dans cet article, semble particulièrement éclairant pour appréhender la créativité suscitée par la maîtrise du fluide électrique. À l'instar d'autres contemporains, on montrera comment penser la mobilité et la motricité à partir de ce cadre conceptuel des fluides lui permet de connecter les uns aux autres des dispositifs techniques différents. En premier lieu, nous aborderons le travail de cet ingénieur militaire introduisant l'usage de l'électricité pour des objets novateurs de mobilité aérienne et sous-marine, puis nous évoquerons sa traduction à la gestion temporelle des flux dans l'espace urbain; enfin, nous nous focaliserons, avec son arrivée chez le constructeur parisien PanhardLevassor, sur son parcours particulièrement fructueux dans l'automobile. 


\section{De la vapeur à l'électricité : mobilité aérienne et sous-marine au service}

Arthur Krebs naît en 1850, à Vesoul (Haute-Saône), dans une famille de petite notabilité provinciale. Les aléas de la guerre de 1870 font de ce passionné de mécanique un Saint-Cyrien plutôt qu'un Polytechnicien. C'est donc dans l'infanterie qu'il commence une carrière d'ingénieur militaire. Son régiment faisant halte à Brest puis à Nantes, il se plaît à visiter les arsenaux des marines publiques et privées, où il perfectionne ses connaissances sur la machine à vapeur. Son régiment venant stationner à Paris en 1876, il fait la connaissance du capitaine Charles Renard, Polytechnicien de trois ans son aîné, qui commence à installer un parc aérostatique à Chalais-Meudon sous la direction du colonel Laussedat ${ }^{6}$. Il obtient son détachement à Chalais et le tandem «Renard et Krebs» qui se met en place va fonctionner jusqu'en 1884 et produire certains résultats. Si l'on peut dire de Krebs qu'il est un «ingénieur-né», alors Renard serait, lui, un «inventeur-né». C'est un esprit en perpétuelle ébullition, servi par une remarquable maîtrise des mathématiques. Depuis ses premiers essais de «plus lourd que l'air» à Arras pendant la guerre, son objectif est de donner à l'homme la maîtrise des airs. Krebs est d'abord un esprit pratique. Il est guidé par la maîtrise de la matière et de l'énergie. Son but est de concevoir des dispositifs qui répondent précisément - c'est-à-dire "par des chiffres certains et vérifiés par l'expérience ${ }^{7} »-$ aux besoins qui leur sont assignés. Pour cela, il souhaite assumer la responsabilité complète des projets techniques qui lui sont confiés.
Les années 1875 sont des années de reconstitution du matériel militaire de l'armée française. Des crédits spécifiques sont votés à cet effet à la Chambre, dont Krebs et Renard bénéficient pour développer un train de ballons captifs mobile en campagne ${ }^{8}$. Depuis le succès des ballons à hydrogène dans les opérations militaires de la Grande armée sous la Révolution, et plus encore avec la récente guerre de 1870 où les ballons libres ont permis de nombreux échanges entre Paris assiégé et la province, l'aérostation fait partie du patrimoine militaire mythique de l'armée française ${ }^{9}$. Un patrimoine qui plaide pour une alliance du militaire avec la science.

Il s'agit maintenant pour les jeunes ingénieurs de concevoir une solution pratique permettant de transporter un ballon captif et ses agrès, son usine de production d'hydrogène et son treuil à vapeur sur le terrain des opérations, le tout tracté par des chevaux. Renard s'attaque au mode de production chimique de l'hydrogène. Krebs s'attelle à la machine à vapeur, au treuil et aux aspects mécaniques du système. Tout est à inventer et à construire sur le parc de Chalais. Krebs prend la direction des ateliers, servis par des soldats des garnisons voisines. Pour actionner les machines de son atelier de scierie, il achète un moteur à gaz Otto construit à Paris par les ateliers de fabrication de machines à bois Périn-Panhard ${ }^{10}$.

Cette mécanique du moteur à explosion passionne Krebs, d'autant plus que le tandem n'a qu'un projet en tête, construire un ballon dirigeable. Tout au 
long du siècle, l'impossibilité de diriger les machines aérostatiques a stimulé un nombre important de projets et de propositions plus ou moins fantaisistes. L'usage des ballons lors du siège de Paris a créé un regain d'attention pour une technologie trop souvent confinée aux démonstrations foraines. Seulement, quelle énergie motrice adopter pour mener à bien ce projet répondant à la demande gouvernementale et militaire? À l'évidence, la vapeur est l'énergie de la puissance, et de la puissance il en faut beaucoup pour réussir à montrer qu'on sait tenir tête à des vents moyens. Le procédé d'un dirigeable à vapeur avait déjà été testé par la grande expérience d'Henri Giffard en 1852, mais de manière encore peu probante. Ici, Krebs et Renard tentent d'améliorer ce procédé en concevant d'abord un dirigeable mû par la vapeur dont ils déposent, en 1878, le projet cacheté à $1^{\prime}$ Académie des sciences ${ }^{11}$.

Pour autant, ils se heurtent inévitablement au même problème qu'ont rencontré toutes les tentatives de directions aérostatiques menées par leurs prédécesseurs, à savoir que l'équation aérostatique entre poids et puissance apparaît rapidement difficile à maîtriser. Le poids du ballon s'accroît par un facteur démultiplicateur avec celui de la puissance du moteur. Il faut à la fois alléger, diminuer les résistances et augmenter le rendement moteur, tout en restant dans des dimensions raisonnables pour des raisons de coût. Krebs et Renard pensent alors utiliser une partie de l'hydrogène emportée dans l'enveloppe pour actionner un moteur à explosion fonctionnant au gaz hydrogène. Mais cette solution audacieuse paraît trop hasardeuse. Elle présente pratiquement le même handicap que la vapeur de placer un foyer de combustion sous une masse énorme d'hydrogène susceptible d'exploser à la moindre étincelle ${ }^{12}$. L'heure est donc aux tâtonnements. Quel fluide moteur se laissera-t-il collecter, transporter, transverser?

Le "fluide électrique ${ }^{13}$ », un phénomène bien connu et central de la science expérimentale du siècle précédent, est de mieux en mieux maîtrisé et s'invite alors comme nouvelle énergie motrice. Renard et Krebs avaient déjà adopté le fil téléphonique enfermé dans le câble de traction pour transmettre les ordres au sol depuis le ballon captif déployé. À cette occasion, ils avaient profité de la venue à Paris de l'Américain Graham Bell pour l'inviter à visiter leurs installations et les conseiller sur leurs projets téléphoniques. Pour produire cette électricité, Krebs avait construit une "machine à lumière» transportable, actionnée par la vapeur.

L'exposition internationale d'électricité de Paris, en $1881^{14}$, est une double révélation pour les deux ingénieurs. D'abord, c'est pour Krebs la révélation que les applications industrielles de l'électricité sont en plein essor. Membre de la Société d'encouragement pour l'industrie nationale, il peut consulter dans son bulletin tous les détails des nouvelles unités à utiliser dans la pratique $^{15}$. Ensuite, c'est la démonstration par les frères Tissandier d'un modèle réduit de ballon dirigeable à moteur électrique. Également héritiers de la tradition des projets aérostatiques de la dernière guerre, les éditeurs de la revue scientifique La Nature ont l'intention de faire rapidement la démonstration en grandeur nature de la possibilité de la navigation aérienne par les voies du plus léger que l'air... et de l'électricité ${ }^{16}$. 
Pour leur dirigeable, Renard et Krebs se décident pour l'électricité, la seule énergie qui ne change pas de poids en cours de route. Renard s'occupe des piles, Krebs du moteur. Après de nombreuses péripéties administratives, les deux ingénieurs militaires obtiennent les crédits nécessaires à leurs expérimentations. Renard invente une pile puissante dite «chlorochromique». Krebs, après des "études préliminaires rationnelles», conçoit un moteur électrique multipolaire léger. L'ensemble est d'un rapport poids-puissance inusité en comparaison de ce que permettent les autres réalisations du moment.

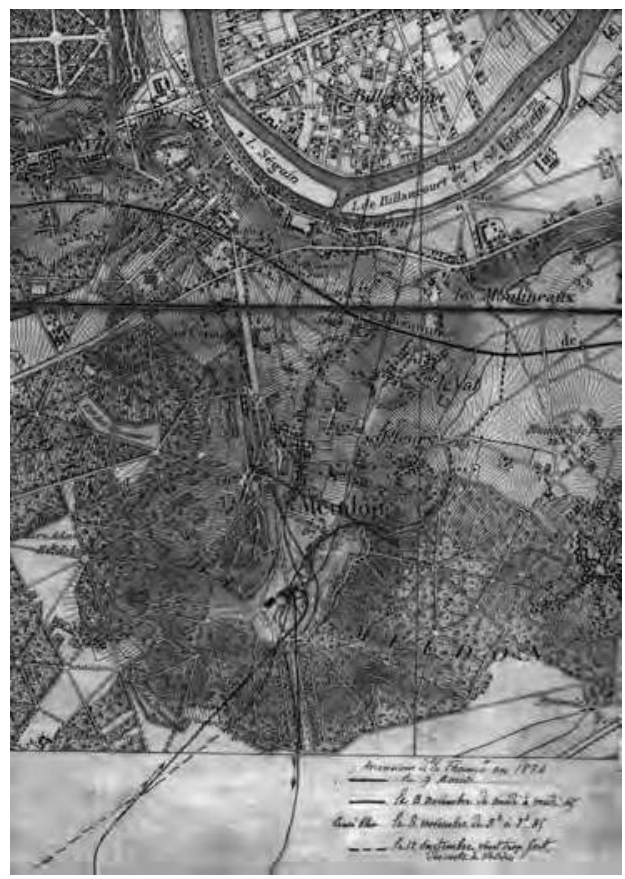

Fig. 2. Carte des quatre trajets réalisés par Krebs avec le dirigeable électrique, extraite de ses archives personnelles (le dirigeable fera trois autres sorties sans lui en 1885) ; les mentions sont de sa main. Ce document montre l'intention de l'ingénieur de prouver la « dirigeabilité » de son engin (archives Krebs).
Finalement, le dirigeable est fin prêt en août 1884. Le 9 août, Krebs prend les commandes, Renard se charge des instruments. Le temps est parfaitement calme. Le navire aérien répond précisément aux manœuvres et parcourt - pour la première fois - un circuit fermé de 8 kilomètres en 20 minutes, au-dessus de Villacoublay. Le fait capital est qu'il soit parvenu à atterrir à son point de départ. Telle était la condition de la réussite de la démonstration. L'année précédente, les frères Tissandier n'y étaient pas parvenus avec leur ballon. La presse française et internationale, ainsi que la presse scientifique, débattent sans fin des conditions de cette réussite inattendue. Le fluide électrique est-il l'avenir de la locomotion aérienne et de la locomotion en général?

Renard et Krebs ont montré ici qu'ils étaient capables de diriger un solide au sein d'un fluide, à l'intérieur de limites de faisabilité très étroites. "Diriger», c'est-à-dire volontairement «imprimer» une force sur l'air, comme on pouvait le dire à l'époque. Cette prise de contact d'un solide sur un fluide, les ingénieurs l'ont obtenue grâce à la maîtrise d'un autre fluide, l'électricité. Collecter l'électricité pour la transporter, c'est la produire et l'emmagasiner. Or, dans le cas de la mobilité, il s'agit pour le mobile autonome de transporter son énergie motrice en même temps que lui. La pile de Renard emmagasine une énergie qui est libérée en cours de route, puis "transversée» dans le moteur électrique de Krebs qui transmet la force produite à une hélice qui elle-même détermine un flux d'air qui, au moyen d'un système de haubanage sophistiqué, permet $\mathrm{d}^{\prime}$ «imprimer la carène aérienne» dans son milieu fluide. L'ensemble est alors «transporté». 
Ainsi, au contraire de la science, comme le montre François Vatin, ce sont les modes opératoires mis en œuvre par l'ingénieur qui définissent précisément l'échelle des fluidités. L'ingénieur Krebs, qui doit maîtriser ces nombreux fluides, est convaincu que le succès de tout projet mécanique tient dans les bons «rapports qui existent entre toutes les dimensions et grandeurs des éléments qui sont réunis ${ }^{17}$ » et finalement dans le rendement total de l'ensemble. L'étude «de l'ensemble et des détails» est une constante des préoccupations de cet ingénieur qui, par ailleurs, est fier de cultiver chez lui «la faculté de bien voir dans l'espace». Cette référence à la proportionnalité des éléments introduit un aspect particulier de la fonction de transversement des fluides soulignée par Leroi-Gourhan. Dans le cas où le fluide est transversé à travers une canalisation, il est possible d'interrompre le débit ou de le régler au moyen d'une écluse, d'un bouchon ou d'un robinet. C'est bien cette caractéristique si propre à l'électricité, dite «dynamique», de circuler nécessairement dans des canalisations qui lui donne ses innombrables possibilités de régulation et qui a si bien permis aux ingénieurs de cette époque de répondre aux besoins de progressivité demandés par leurs commanditaires.

Or, il ne suffit pas, pour l'ingénieur, de faire la preuve de la validité scientifique d'un artefact. L'objet lui-même doit tisser un réseau de relations autour de lui pour prendre la force de l'autorité au sein de son milieu ${ }^{18}$. L'aéronaute Wilfrid de Fonvielle, dans son livre de $1884^{19}$, pose ainsi la question de la légitimité de cette «victoire» pour les deux ingénieurs militaires. Il minimise la portée tech- nique de l'exploit. Il mentionne ensuite l'antériorité du brevet de dirigeable électrique déposé par les frères Tissandier. Enfin, il met en cause le financement public de l'expérience. Pour lui, c'est la connaissance des courants aériens qui est l'avenir de la navigation aérienne:

«N'y aurait-il pas, en effet, folie à dépenser une force motrice considérable, à lutter contre un vent d'une certaine violence quand il suffirait de jeter un sac de lest pour rencontrer à quelques centaines de mètres le courant favorable, qui porterait vers le but désiré avec une vitesse beaucoup plus grande que celle qu'on pourrait se procurer en usant, soit du combustible, soit de l'électricité.»

En écho à la première nautique aérienne développée de manière très fine dès 1785 par l'ingénieur et mathématicien Meusnier de la Place et qui considérait que celle-ci consistait à «faire un emploi éclairé des vents", il s'agit là aussi de se laisser porter par les forces naturelles et non de maîtriser les fluides pour mieux les transformer ${ }^{20}$. L'idée même d'automobilisme et de ballon dirigeable électrique est ici sans objet. Mais, si on lit bien Wilfrid de Fonvielle, la véritable traduction $\mathrm{du}$ ballon de Renard et Krebs est à chercher du côté de l'image de son groupe social, méprisé pendant des décennies par les détracteurs - y compris scientifiques - de tous bords. C'est au compte du travail ancien de son réseau social sur le sujet de la dirigeabilité aérienne qu'il reprend l'effet psychologique positif produit dans les esprits par la démonstration du dirigeable militaire: 
«Si elle a une importance capitale, c'est qu'elle a permis de montrer aux ignorants et aux sceptiques de parti pris que la recherche de la direction des ballons ne doit pas être confondue avec la quadrature du cercle ou le mouvement perpétuel. »

Même si la solution proposée par Renard et Krebs pour la direction des ballons est d'abord un ensemble de choix pragmatiques parmi ceux proposés auparavant ${ }^{21}$, mis en œuvre avec une rigueur toute scientifique, le recul du temps montrera que le succès du dirigeable militaire était notoirement dû à son hangar spécialement construit pour le remiser tout gonflé et lui permettre ainsi d'attendre la météo favorable pour effectuer une sortie sans danger ${ }^{22}$. L'électricité fut donc ici essentiellement médiatrice des rêves de modernité et d'autonomie d'une France en reconstruction.

La relation analogique entre l'air et l'eau connaît un renouveau conceptuel et technique particulièrement fructueux dans le contexte technique et politique du dernier tiers du XIX ${ }^{\mathrm{e}}$ siècle $^{23}$. En 1885, la loi sur la «liberté de fabrication des engins de guerre» autorise les chantiers navals privés à postuler aux appels d'offres de la Marine. Dupuy de Lôme, académicien et ingénieur du génie maritime, lui-même auteur d'un grand essai de dirigeable financé par la III ${ }^{e}$ République naissante en 1872, obtient avec son gendre Gustave Zédé, administrateur des Forges et Chantiers de la Méditerranée, l'attribution d'un marché de construction d'un "torpilleur sousmarin électrique». Ils proposent alors à Krebs, désormais reconnu comme «élec- tricien", de prendre en charge la conception du moteur électrique et de toute la partie électrique et mécanique du projet de sous-marin. C'est une équipe dispersée qui doit le mener à bien. Krebs est alors en poste au régiment des sapeurspompiers de Paris, où il est chargé de mettre en place une nouvelle organisation et un nouveau matériel. Gaston Romazzotti, neveu de Zédé, est l'ingénieur du génie maritime chargé de la construction du sous-marin à l'arsenal de la Marine de Toulon. Le moteur électrique de Krebs sera construit aux chantiers de la Méditerranée au Havre.

Tout est électrique dans ce navire qui doit «s'affranchir» de la surface, suivant le terme consacré. Une batterie d'accumulateurs rechargeable occupe la plus grande place. Des "caisses d'eau» et des réservoirs d'air comprimé occupent l'avant, le centre et l'arrière. Le moteur ferme ensuite tout l'arrière. L'éclairage et les organes de manœuvres (pompes et servo-moteurs) sont électriques. Un périscope et un gyroscope, tous deux électriques, permettent la navigation en surface et en plongée. À nouveau, la réussite de cet engin mécanique novateur passe par la maîtrise de nombreux fluides et par leur bonne coordination mutuelle. Faire plonger le Gymnote, qui, au port, ne laisse émerger que sa plateforme, consiste à mettre en marche le moteur à petite vitesse tout en remplissant d'eau des réservoirs et en chassant l'air qu'ils contiennent, d'abord ceux de l'avant, puis, progressivement, ceux de l'arrière. Il faut simultanément faire agir les ailerons qui, sous la vitesse acquise, permettent de modifier la profondeur et la direction. Ensuite, un pendule électrique règle «automatiquement» l'as- 


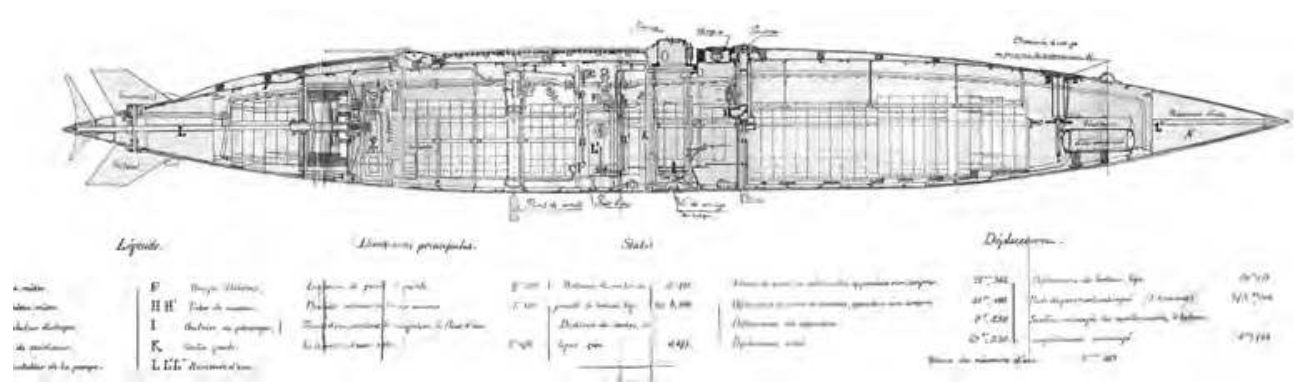

Fig. 3. Coupe du sous-marin Gymnote montrant le «tuyautage» de ses fluides : eau, air, électricité (annexes de l'Étude sur les bateaux sous-marins par le lieutenant de vaisseau Darrieus, avril 1895, document héliogravé, Archives de la Marine, 6W6).

siette pour maintenir une immersion constante.

Les problèmes sont nombreux et difficiles car le navire est de dimensions très réduites. Les premiers essais du sousmarin Gymnote sont pourtant concluants fin 1888, puis fin 1889. Son rayon d'action étant très limité, il s'agit d'un engin expérimental n'ayant aucune valeur militaire. Il porte pourtant à son actif le premier forcement de blocus en rade de Toulon en 1890, qu'il n'a pu réussir que grâce à son gyroscope électrique ${ }^{24}$. Il subira de nombreuses transformations et servira de navire de formation pour les élèves sous-mariniers jusqu'en 1907. Assez mal reçu par certains milieux de la Marine $^{25}$, il décidera cependant le ministère à continuer dans la voie de la création de la première flotte sous-marine française.

Avec le dirigeable électrique, nous parlions d'un "mobile autonome» sous la forme d'un ensemble de fluides collectés, transportés, transversés. Avec le sous-marin Gymnote, l'aspect «transport» des fluides devient prépondérant par la nécessité d'une coupure totale du mobile à l'égard de la surface. Une contrainte que la vapeur ne peut satisfaire. À nouveau, c'est l'électricité qui est l'agent validateur de ce principe d'indépendance.

Le sous-marin a en effet une particularité bien à lui, qui prouve cette réelle indépendance: quand il a plongé, il disparaît aux yeux des observateurs restés sur la berge. Quelle meilleure preuve de son autonomie? Si cet engin est capable de se soustraire aux regards, c'est bien qu'il est doué d'une vie intérieure, qu'il est un être, un être invisible. Et l'électricité qui, par essence, est invisible, habite ce vaisseau comme le ferait une âme dans un corps. En un mot, l'idée du fluide électrique comme vitale, pensée qui a animé notamment tout le courant de l'électricité animale, fait de la maîtrise de ce fluide une manière d'échapper à la matière et au temps. 


\section{Le fluide-temps : pensée de l'organisation et de la circulation}

Cette approche conceptuelle et pratique singulière donne lieu à toutes sortes d'applications dans le contexte de l'émergence de la ville des flux et des réseaux au $\mathrm{XIX}^{\mathrm{e}}$ siècle $^{26}$. La réorganisation du service d'incendie de la Ville de Paris est une affaire de «science municipale $»^{27}$. Le député socialiste et conseiller municipal de Paris, Léon Donnat, fait la promotion de l'idée de "politique expérimentale» qu'il justifie notamment en affirmant que «la France, ayant perdu ses traditions, ne peut demander qu'à la méthode expérimentale une politique nouvelle ${ }^{28} \gg$. Autrement dit, la jeune République française ne peut espérer approcher les vérités qui sont les siennes propres qu'au travers d'une nouvelle rationalité partagée, une rationalité où les ingénieurs ont la part belle. Le fluide de l'unité nationale semble ici passer par la vérité scientifique et technique qui elle-même doit montrer qu'elle sait maîtriser les fluides naturels ${ }^{29}$.

Dans cet esprit, Léon Donnat propose, en $1887^{30}$, la mise en place d'un comité de perfectionnement du régiment de sapeurs-pompiers de Paris destiné à examiner les améliorations à apporter à ce service. Le préfet Léon Bourgeois crée ce comité en réunissant des membres du Conseil municipal, des ingénieurs des services municipaux et des officiers du régiment. Les récents incendies dans les théâtres ${ }^{31}$ ont également motivé la création de ce comité qui a pour mission de proposer les crédits nécessaires à la réorganisation complète du service d'incendie. Dès 1881, le colonel Paris, Polytechnicien et chef de corps des pompiers de Paris, avait fait l'inventaire des réformes à effectuer sous la forme d'un livre intitulé Le feu en France et en Amérique $^{32}$. C'est la science municipale qui, par la comparaison internationale rationnelle qu'elle impose, lui permet de justifier ses propositions de réorganisation auprès des édiles parisiens et de son administration préfectorale de tutelle ${ }^{33}$.

$C^{\prime}$ est dans ce cadre que le capitaine Krebs est amené à parcourir l'Europe jusqu'à Moscou et les États-Unis à deux reprises. Il visite les services d'incendie de ces différents pays, mais également nombre d'usines où il peut s'entretenir avec les ingénieurs. Il est, par exemple, très probable que son adoption, dès 1886, des inducteurs électriques à empilement de tôles soit le résultat d'une de ses conversations avec un ingénieur américain lors de son voyage de 1885 .

Pour le pompier, le feu est un fluide qu'il s'agit de contenir, de circonscrire, pour mieux le maîtriser. L'eau est cet autre fluide qui, rationnellement canalisé, contenu et déversé, est le principal agent de lutte contre l'incendie. Mais, cette fois, la mission de l'ingénieur consiste à concevoir une organisation qui unisse dans une même vérité - un même fluide - le matériel et l'humain. À partir de toutes les variables, très hétérogènes, recueillies à l'étranger et par l'analyse minutieuse de l'existant parisien, il élabore un plan de réforme de grande envergure, forgé pour un contexte urbain nouveau dont la mise en œuvre doit s'étaler dans le temps en raison de l'importance des investissements à réaliser. Les premières variables 
qui s'imposent sont endogènes au corps, à son statut militaire et à sa mission très spécifique qui exige une disponibilité permanente.

Sans entrer ici dans les détails de ce plan, énumérons ses principales variables techniques. L'extension du nombre des "bouches d'incendie» autorise le remplacement des pompes à bras par les dévidoirs de tuyaux. Le téléphone permet de meilleures communications entre les centres de secours, et diminue les temps d'intervention en permettant au public de signaler l'incendie au plus près du sinistre. La construction de casernes centrales équipées d'un matériel puissant, au lieu de postes disséminés et sous-équipés, permet une concentration des forces, la traction hippomobile, et non plus humaine, du matériel, un logement décent et une meilleure formation du personnel, ainsi que la suppression des surcharges de travail. La standardisation des matériels et des accessoires permet leur interchangeabilité, simplifie leur manœuvre et leur maintenance et réduit leur coût. La conception d'un matériel nécessitant moins de servants permet d'utiliser plus de personnel à la lutte contre le sinistre. Citons l'exemple du compresseur hydraulique qu'il invente. La pompe à air à bras de 250 kg, utilisée pour les feux de cave, nécessitait quatre sapeurs pour sa manœuvre. Le compresseur à effet venturi, qui se branche sur les bouches d'incendie, ne demande qu'un servant lors de sa mise en marche et ne pèse que $15 \mathrm{~kg}$. Une vraie démonstration des possibilités qu'offre la maîtrise du fluide-eau.

Dans son plan de réorganisation, Krebs n'évoque aucune mise en œuvre d'un quelconque modèle de flux. Pourtant, le parcours d'un flux définit une spatialité et une temporalité et, comme tel, détermine un contexte opératoire ayant valeur de système. Si le modèle du flux ne construit pas à proprement parler le système, on peut penser qu'il le produit discrètement par implication, en l'induisant par son usage. Or, nous avons vu que Krebs a clairement l'intention de mettre en place un «programme» ordonnanceur des rôles et des comportements, à destination des «hommes» et du matériel, qui mette en œuvre des flux physiques et organisationnels de façon généralisée. Il en résulte un système orchestrateur de flux, lui-même orchestré par ses propres flux. Un tel système pouvant tendre vers l'autoréférentiel du fait qu'il exige une disponibilité permanente de l'ensemble de ses flux, demande un mode de régulation rigoureux pour être maîtrisé. La variable «temps d'intervention» doit muter quant à sa mise en œuvre.

L'ingénieur ne doit plus seulement maîtriser les fluides matériels (la vapeur, l'hydrogène, l'électricité, l'eau), il doit également maîtriser le fluide-temps, aussi invisible que le fluide électrique, mais bien plus difficile à contenir, transporter, transverser. La réponse que Krebs propose pour résoudre l'équation porteuse de toutes les variables qu'il a identifiées se nomme «vitesse». Ce n'est plus la position de proximité physique du pompier près du sinistre qui va lui assurer son efficacité maximum, c'est la vitesse avec laquelle l'organisation globale du service saura faire parvenir sur les lieux du sinistre la juste quantité de matériel et de personnel, d'où que proviennent ce matériel et ce personnel. Si la proximité fige l'organisation et limite les possibilités d'adaptation, les capacités 
de la vitesse sont, quant à elles, potentiellement illimitées.

Krebs propose alors de diviser Paris en vingt-quatre secteurs d'intervention ${ }^{34}$ dont les surfaces sont désormais déterminées par les vitesses ${ }^{35}$ :

«[...] les surfaces des périmètres défendus chacun par un poste sont proportionnelles au carré des vitesses avec lesquelles ces postes peuvent se transporter et, comme conséquence immédiate et très importante, le nombre des postes à placer est inversement proportionnel au carré de ces mêmes vitesses de déplacement. Ainsi, un système de transport qui permettrait d'aller deux fois plus vite qu'un autre exigera quatre fois moins de postes pour arriver dans le même temps ${ }^{36}$.»

Cette politique implique un matériel adapté à la vitesse, des moyens de

\section{La cause automobile}

En 1894, la première course d'automobile, Paris-Rouen, médiatisée par la presse et les notabilités parisiennes, est à l'origine d'un véritable empressement à l'égard de l'automobilisme en France ${ }^{39}$. Le service privé de location de chevaux étant très coûteux pour le budget de la Ville, Krebs propose alors de mener des essais de traction mécanique en achetant un moteur à pétrole Daimler construit à Paris par les usines Panhard-Levassor, anciennement Périn-Panhard. Il obtient des crédits pour construire une "petite voiture d'expérience» qui permette de faire une démonstration pratique. communication déployés en «réseau» et fonctionnant «à la vitesse de l'électricité», une formation technique poussée des personnels, ainsi qu'une discipline militaire. François Vatin a montré ${ }^{37}$ combien, entre les mains de l'organisateur ou du gestionnaire, le modèle du flux permet de penser l'unité de l'ordre et du désordre et renvoie au problème du contrôle des "masses turbulentes »: «La fluidité désigne clairement l'idéal du gestionnaire: canaliser, homogénéiser pour contrôler, régler et diriger les mouvements.» La fluidité, c'est aussi gérer les flux et les circulations accrues à mesure que la métropole s'accroît pour atteindre le million d'habitants. C'est dans ce contexte que se comprennent l'intégration et l'usage d'une pensée de la mobilité qui met l'accent sur de nouvelles techniques motorisées incarnées par l'apparition de l'automobile ${ }^{38}$.

C'est un concept complet d'automobile que l'ingénieur crée ici: esthétique, confortable, sûre et "simple de manœuvre». L'esthétique est empruntée à la voiture à cheval. La "stabilité de route» résulte de la géométrie du train avant - l'angle de chasse - empruntée à la bicyclette dite «moderne». Le confort est assuré par la suspension des organes mécaniques, suivant le principe que «trois points suffisent pour déterminer un plan». La simplicité de manœuvre, quant à elle, est empruntée à l'électricité. L'ingénieur a en effet imaginé une boîte de vitesses électro-magnétique où 
les changements de vitesse s'effectuent à l'aide d'un simple commutateur.

Cette solution technique convainc Émile Levassor, le vainqueur des premières courses automobiles, qui croit en son avenir pour le marché des taxis urbains. En 1896, il achète la licence du brevet ${ }^{40}$ et demande à l'inventeur d'en suivre la construction dans son usine. Quelques mois plus tard, Levassor décède et René Panhard demande à Krebs de prendre sa succession. Celui-ci accepte et quitte l'armée. Une nouvelle société anonyme vient de se créer. Le "Commandant Krebs» en devient le directeur d'usine.

Avec son arrivée chez PanhardLevassor, Krebs est bel et bien devenu «pétroliste». Or, au tournant des années 1900, le moteur à pétrole est une mécanique bien peu sociable. Il manque de puissance pour se rendre utile dans le transport de charges lourdes, mais il l'est suffisamment pour écraser force gens et animaux en campagne. Il est capricieux et demande un entretien rigoureux. En ville, il est bruyant, dégage des odeurs fort désagréables et, à l'arrêt, il est pris de secousses insupportables. Enfin, la concurrence entre les constructeurs étant rude, il se démode très rapidement. Tous ces inconvénients sont inconnus de la traction électrique.

Pour comprendre ce qui peut apparaître comme une volte-face, nous pouvons écouter les justifications de $l^{\prime}$ «expert» lors du procès Selden, à NewYork, en $1906^{41}$, justifications qui prennent d'abord la forme d'une chronologie des événements. En 1867, le jeune homme de dix-sept ans, alors élève en classe de Math-Sup au lycée de Besançon, se rend à Lons-le-Saulnier pour assister à la démonstration par $\mathrm{M}$. Lotz de sa «voiture sans chevaux» à vapeur, tant à titre d'essai que de publicité. L'année suivante, il dessine un «projet de voiture automobile» à vapeur dont la longue colonne de direction inclinée suggère une indéniable intention de vitesse ${ }^{42}$. L'année de l'exposition universelle de 1878 à Paris, Krebs, comme vu plus haut, achète un moteur à gaz Otto chez Périn-Panhard pour son atelier de scierie à Chalais-Meudon. Il effectue alors des recherches en direction des moteurs légers avec combustion à "pression constante», dont l'allumage peut s'effectuer sans flamme. Ne parvenant pas à obtenir un rapport poids-puissance suffisant, il décide de se tourner vers l'électricitét3.

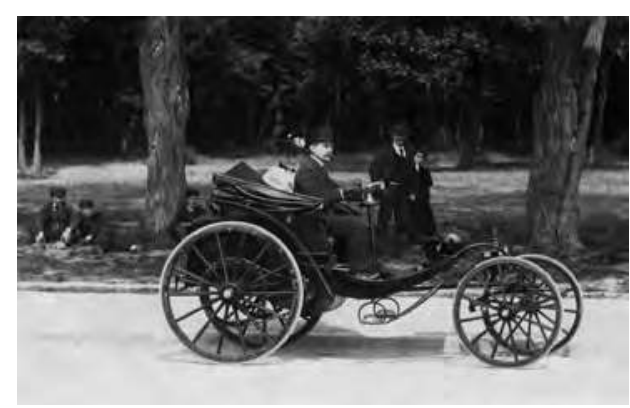

Fig. 4. La voiture du brevet Krebs au bois de Boulogne. Antoine, le jeune fils, prend en photo son père et sa mère à bord de la voiture construite chez les pompiers ; on aperçoit sous le guidon le commutateur de la boîte de vitesse électromagnétique (archives Krebs).

À partir de 1884, date à laquelle il entre chez les pompiers, il suit les progrès des «petits moteurs à pétrole» de l'Allemand Gottlieb Daimler. À l'exposition universelle de 1889, il peut observer Daimler, Panhard et Levassor faire naviguer leurs canots à pétrole sur la Seine. Levassor faisant peu après l'adaptation de ce moteur sur un véhicule, il peut suivre à 

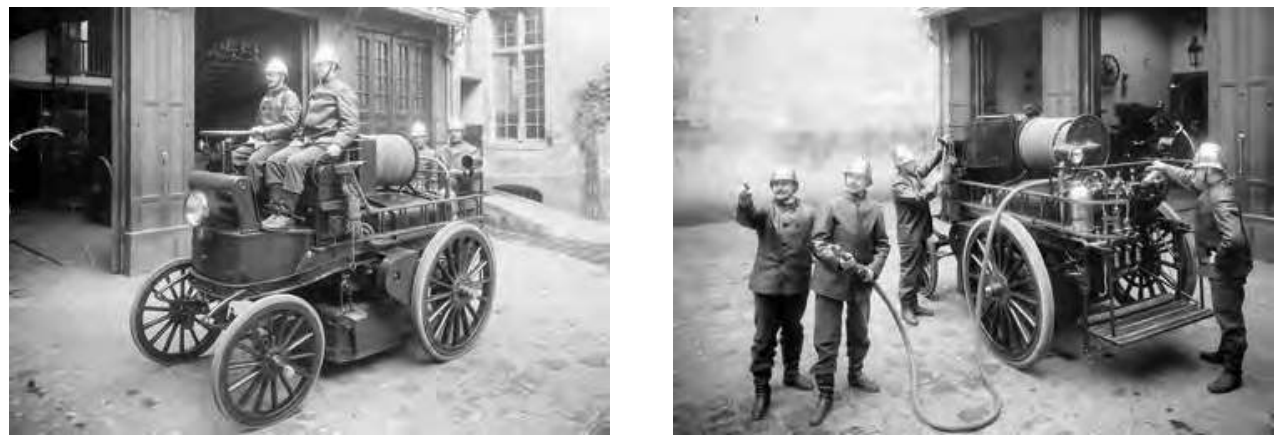

Fig. 5 et 6 . Le fourgon d'incendie électrique, de face et d'arrière. Une vraie modernité se dégage de ce véhicule avec sa compacité, son phare à l'avant et son esthétique fort éloignée de celle des voiture hippomobiles (http://www.culture.gouv.fr/public/mistral/memsmn_fr).

Paris l'évolution des modèles PanhardLevassor, commercialisés à partir de 1891. Mais l'événement déterminant qui mobilise toutes les consciences curieuses de mobilité a bien lieu en 1894. Le 18 juillet de cette année, tôt le matin, le capitaine-ingénieur des pompiers est présent au départ de la première course automobile, Paris-Rouen, organisée par le journal Le Matin. Il peut constater la multitude des motorisations présentes, qu'elles soient électriques, à vapeur, à pétrole... ou appartenant à l'un des vingt-trois autres systèmes proposés ${ }^{44}$. $\mathrm{Au}$ travers des termes du règlement, qui stipulait que «toutes les qualités des voitures seront alors considérées: vitesse, stabilité, économie, sécuritét ${ }^{45}$ ", on cherchait «la petite voiture pour le touriste et le commerçant, pratique à manier, élégante, etc.». Face à la bicyclette et à la vapeur, ou même au cheval, l'argument premier en faveur du nouveau moteur à pétrole n'est ni la vitesse ni la puissance ${ }^{46}$, mais bien le concept de «véhicule autonome ${ }^{47}$ ». Auprès des organisateurs du concours, c'est bien le petit moteur à pétrole qui fait l'unanimité comme moteur de l'avenir, par sa capacité potentielle à satisfaire à toutes les exigences requises.

Dans ce contexte, nous ne sommes pas étonnés de voir l'ingénieur Krebs, passionné de mécanique automobile depuis son plus jeune âge, commencer à étudier non seulement la technique du moteur à pétrole, mais aussi son application à la locomotion automobile. Cette phase d'étude s'achève en 1896, quand il dépose son brevet de système automobile en France et en Angleterre et quand, l'année suivante, il succède à Émile Levassor comme directeur des usines Panhard-Levassor. Il obtiendra bientôt l'adoubement formel du maître, quand, en octobre 1897, il sera reçu par Gottlieb Daimler, chez lui à Canstadt, en Allemagne ${ }^{48}$.

Dès 1895, Krebs était arrivé à la conclusion que le moteur à pétrole n'avait pas une puissance suffisante pour tracter les véhicules d'incendie ${ }^{49}$ et que son démarrage restait peu fiable. Il s'était donc, à nouveau, tourné vers l'électricité et avait conçu un "premier secours» tout électrique, composé de trois voitures - une pompe, un fourgon d'agrès et une grande échelle - qui entrera en service en 1899. 
Des véhicules très compacts qui tirent toutes les conséquences des avantages de l'électricité; ils démarrent instantanément et leur autonomie, à l'échelle d'un secteur d'intervention parisien, permet à la pompe d'emporter une provision d'eau, utile pour attaquer le feu pendant qu'on établit les lances sur les bouches $\mathrm{d}^{\prime}$ incendie $\mathrm{e}^{50}$. Ce n'est en effet qu'à partir de 1910 que le pétrole viendra définitivement motoriser le matériel du corps.

Pour le nouveau directeur d'usine, de nouvelles équations sont à résoudre dont certaines relèvent de la nouvelle «science industrielle» promue à cette époque par Henry Le Chatelier ${ }^{51}$. L'ancien militaire doit montrer à ses commanditaires $\mathrm{du}$ conseil d'administration qu'il est l'homme capable d'assurer la bonne gestion de leur capital. Un capital social fait de numéraire, de bâtiments d'usine, de personnel qualifié, de machines-outils, de brevets, mais surtout d'une image d'autorité historique, celle de l'entreprise par qui l'automobile pratique est née.

À la confluence de toutes ces variables socio-techniques, l'ingénieur va donc se faire le promoteur de la «cause automobile» et du moteur à pétrole et composer une politique technique tendant à consolider "l'héritage acquis", tout en multipliant les innovations. L'une des composantes de cette politique va consister à maîtriser les fluides en jeu et notamment à tenter d'associer le pétrole avec l'électricité.

Pour cela, il va d'abord s'attacher à contrôler efficacement le dosage du mélange air-essence admis au moteur. Son carburateur automatique de $1902^{52}$ est ainsi sa réponse mécanique au besoin d'accélération progressive demandée par le propriétaire d'automobile, mais également sa solution au besoin d'utilisation rationnelle de l'essence par un moteur à pétrole. Puis il va breveter un système électro-magnétique de contrôle des soupapes d'admission qui sert à la régulation $\mathrm{du}$ régime moteur ${ }^{53}$. Enfin, il va investir le complexe domaine de l'allumage électrique du mélange ${ }^{54}$. Il s'agit ici d'une véritable hybridation des techniques, au point qu'on a pu dire à l'époque que c'est l'électricité qui avait «sauvé» le moteur à essence dans la confrontation qui l'opposait alors aux moteurs à vapeur et électriques ${ }^{55}$.

L'exposition universelle de Paris de 1900, où l'électricité est sacrée reine, donne à Krebs l'occasion de poursuivre dans la voie de l'hybridation, d'autant que le belge Jenatzy vient de montrer, en 1899, avec son automobile la Jamais contente, que l'électricité est capable de vitesses exceptionnelles. Sur le stand de la société autrichienne de matériel électrique Lohner, il découvre le véhicule présenté par l'ingénieur Porsche. C'est un véhicule mixte pétrole et électrique, dit «électromobile». Un petit moteur à pétrole de Dion produit l'électricité qui est fournie aux moteurs électriques placés à l'intérieur des roues du véhicule. Porsche justifie cette disposition par le bon rendement mécanique de ce type de transmission, un rendement meilleur que le «tout mécanique». En effet, dès cette époque, on sait fabriquer sans difficulté des moteurs électriques dont le rendement est supérieur à $90 \%$, alors qu'il ne faut pas espérer dépasser un rendement de $12 \%$ avec un moteur à pétrole destiné à l'automobile ${ }^{56}$. De plus, la solution proposée par Porsche élimine tous les organes de transmission mécaniques, consommateurs de puis- 
sance motrice, l'embrayage, la boîte de vitesses, les joints de cardan, le différentiel, les chaînes et leurs pignons.

Cette équation est en parfaite osmose avec les idées de Krebs qui n'a de cesse de mesurer l'efficacité de la diffusion du «fluide mécanique». À cet effet, il convoque à nouveau l'électricité comme agent validateur du rendement mécanique et invente - ou réinvente ${ }^{57}-\mathrm{le}$ «dynamo-dynamomètre» ou «frein dynamométrique électrique $»^{58}$. Il s'agit pour lui de substituer au frein à friction mécanique de Prony un frein à friction électro-magnétique qui permette de mesurer la puissance des moteurs et, par extension, le rendement de tout système mécanique. Basé sur les mêmes formules mathématiques, il autorise une mesure précise sur une durée indéfinie ${ }^{59}$. À partir du brevet Lohner-Porsche acquis par Panhard, Krebs conçoit, en 1902, un véhicule où pétrole et électricité sont en relation quasi symbiotique, où l'électricité assure une autorégulation généralisée et où le mécanique est réduit à son minimum. Le démarrage et l'allumage du moteur à pétrole, ainsi que l'éclairage sont électriques. Les moteurs électriques intégrés dans les roues avant servent également de freins électro-magnétiques, en complément des freins mécaniques à l'arrière. La dynamo génératrice sert de volant moteur, mais surtout une certaine mobilité donnée aux inducteurs lui permet de commander en retour le régu- lateur électro-magnétique des soupapes $\mathrm{d}^{\prime}$ admission en fonction de la charge du moteur ${ }^{60}$.

Les résultats de l'investissement de longue date de cet ingénieur sur le domaine de la mobilité, des fluides et de l'électricité sont tangibles même si la nature de ces apports possède elle-même cette propriété de fluidité qui les rend si miscibles dans la complexité des objets produits. On peut en effet noter que les objets techniques conçus par Krebs répondent étonnamment bien à la définition du processus de "concrétisation" proposé par Gilbert Simondon ${ }^{61}$. Mais le fait le plus caractéristique de ce parcours est son bouclage unique sur la question de la mobilité et de sa motorisation. Nous avons vu le jeune ingénieur s'interroger sur la voiture à vapeur de Lotz. Puis, nous l'avons vu adopter le moteur électrique pour parvenir à démontrer la dirigeabilité des ballons et des sous-marins. Nous l'avons vu ensuite embrasser le mouvement de la voiture à pétrole en France et breveter sa première voiture automobile. Or, dès la domination du moteur à pétrole acquise sur ses concurrents électriques ou à vapeur, on voit Krebs livrer à l'armée des moteurs Panhard-Levassor pour les dirigeables militaires Lebaudy et les sous-marins de la classe Nä̈ade. Enfin, le rapport poids-puissance de ces moteurs s'améliorant sans cesse, il conçoit des moteurs d'aviation à partir de 1910. 


\section{Conclusion : la fluidité comme modèle épistémologique}

Pour conclure, nous avons vu combien, au cours de ces années post-1870, la mobilité était une résultante des premiers succès des ingénieurs dans leurs efforts pour maîtriser d'abord les fluides naturels comme l'eau, puis est traduite dans un ensemble plus vaste, dans leur capacité à répondre aux besoins des milieux socio-techniques existants quant à leurs attentes locales. L'électricité, par sa facilité de circulation et ses capacités de régulation, a rapidement pris le rôle d'agent validateur des hypothèses mécaniques. Avec le téléphone, elle a montré l'influence de la vitesse sur les organisations. Avec l'automobilisme, c'est le pétrole qui s'est imposé comme agent de vitesse et d'autonomie des mobiles, tandis que l'électricité se voit confirmée dans son rôle de régulateur et de mesure des fluides.

Le caractère spécifique $\mathrm{du}$ modèle épistémologique introduit par le modèle du flux et l'idée du fluide a permis d'accompagner la pensée de l'ingénieur depuis la vapeur jusqu'au pétrole, en passant par l'hydrogène, l'électricité, l'eau, le feu, etc. Selon Jean Mathiot, «le statut métaphorique du flux se perd quand il se réalise, soit dans la chose fluide, soit dans la forme fluide ${ }^{62} \gg$. Il s'ensuit la nécessité permanente de recomposer l'idée de fluide suivant les nombreux besoins techniques des ingénieurs. Ne peut-on voir dans cette nécessité de recomposition l'essence de la modernité qui s'attache à nos sociétés industrielles, jusqu'au caractère majeur que conserve la fluidité comme structurante des sociétés post-modernes ${ }^{63}$ ?
À la fin de ce siècle, les travaux de Branly et Marconi sur la radioélectricité renforcent les liens entre le monde de l'invisible et le fluide électrique qui prend alors pratiquement le statut d'«éther», cet invisible qui attirait tant l'attention des scientifiques les plus rationnels de cette époque. Le commandant Krebs ne fit pas exception quand il participa, en 1908, aux côtés de Marie Curie (physicienne de la radioactivité naturelle) et d'autres personnalités du monde scientifique $^{64}$, à des séances de spiritisme organisées par son ami Arsène d'Arsonval, médecin, physicien des courants électriques à haute fréquence et académicien, séances destinées à comprendre et mesurer scientifiquement le phénomène du fluide spirite ${ }^{65}$. Une époque, encore, où Albert Einstein propose, en 1905, d'abandonner cet «éther » du xIx ${ }^{e}$ siècle au profit d'un très heuristique concept de "continuum espace-temps", où la vitesse de la lumière, conçue comme un flux de particules, a valeur d'étalon indépassable dans le cadre d'un nouveau "principe de relativité ${ }^{66}$. Plus que jamais, l'idée de fluide permet de décrire le monde objectif relativement au sujet observateur. Une époque, enfin, où l'on voit le commandant Krebs mettre en œuvre l'idée d'«organisation scientifique du travail» proposée par Taylor, avec l'aide de son beau-frère Charles de Fréminville qui ne cesse de militer pour une rationalisation du travail «sans heurts et sans secousses» par $l^{\prime}$ «étude des temps» jusqu'au niveau des opérations élémentaires de travail ${ }^{67}$. Cette rationalisation de l'écoulement matériel 
$\mathrm{du}$ fluide-temps, telle fut bien encore l'idée de Henry Ford quand il conçut sa «chaîne mobile» de production en 1913.

\section{Notes}

1. Sadi Carnot, Réflexions sur la puissance motrice du feu et sur les machines propres à développer cette puissance, Paris, 1824; Robert Fox, CARNOT, Sadi. Réflexions sur la puissance motrice du feu, édition critique..., Paris, Vrin, 1978; idem, The caloric theory of gases from Lavoisier to Regnault, Oxford, Clarendon Press, 1971.

2. Jean Mathiot, «Humeurs fluides. Réflexions sur un modèle épistémologique», Espaces et sociétés, $\mathrm{n}^{\circ} 43$, Flux, espaces, sociétés, Paris, Anthropos, 1983, p. 12 et 51.

3. André Leroi-Gourhan, Évolution et techniques. L'homme et la matière, Paris, Albin Michel, 1943-1971, p. 297.

4. S. Carnot, Réflexions sur la puissance motrice du feu..., op. cit., p. 28.

5. J. Mathiot, "Humeurs fluides...», op. cit., p. 54 .

6. Claudine Fontanon et André Grelon. Les professeurs $d u$ Conservatoire national des arts et métiers. Dictionnaire biographique, Paris, Institut de recherches pédagogique-CNAM, 1994: «Aimé Laussedat (Colonel) (1819-1907) [Polytechnicien], professeur de géométrie appliquée aux arts (18731896), directeur du Conservatoire (1881-1900)»; Revue générale des sciences pures et appliquées, Paris, 15 mai 1907: «Nécrologie. [...] De 1872 à 1879, le colonel Laussedat se consacra à l'étude des divers moyens de communication par voie aérienne (télégraphie optique, éclairage à distance, pigeons voyageurs, aérostation militaire) avec le concours de la Commission où il eut le mérite de faire entrer des hommes comme le colonel Mangin et le capitaine Renard.»

7. Hervé Mangon, «Sur un aérostat dirigeable. Note de MM. Ch. Renard et A. Krebs», Comptes rendus des séances de l'Académie des sciences, Paris, Gauthier-Villars, 18 août 1884.

8. Le fonds des archives Renard est disponible aux archives du Génie, à Vincennes.

9. Patrice Bret, L'État, l'armée, la science: l'invention de la recherche publique en France (1763-1830), Rennes, Presses universitaires de Rennes, 2002; Marie Thébaud-Sorger, Une histoire des ballons. Invention, culture matérielle et imaginaire, 1783-1909, Paris, Éditions du Patrimoine, 2010, p. 28-32 sur la question patriotisme et $\mathrm{p}$. 127-129.

10. Arthur Krebs est entendu au procès Selden, à New York, en tant qu'expert, du 31 octobre au 21 novembre 1906; National Archives and Records Administration, Office of regional records service, SRG 21, records of the district courts of the United States, U. S. Circuit Court, Southern district of New York, Equity case 8616: Electric Vehicle Co. \& George B. Selden vs S. A. des Anciens Établissements Panhard et Levassor and André Massenat. Arthur Constantin Krebs Testimony, p. 66: «A116. [...] During that year [1878] while at the aeronautic station I bought and accepted, at the shops of that firm [Périn Panhard et Cie], a two horse power Otto four cycle engine for installation at the balloon sewing shop, which installation I referred to at the beginning of these interrogatories.», http://en.wikipedia.org/wiki/ File:1906_Arthur_Constantin_KREBS_testimony_ during_the_Selden_case.pdf.

11. Ils déposent également un brevet $n^{\circ} 124.078$ du 25 avril 1878, aux noms de de la Haye, Renard et Krebs: Générateur de vapeur à circulation directe, dit générateur Renhaye.

12. Renard construira en 1891 un moteur mixte, à l'hydrogène et au benzol, mais celui-ci cassera au banc d'essai sous les coups de ses vibrations excessives. Très inspiré du moteur à vapeur, il est entreposé dans les réserves du musée de l'Air et de l'Espace du Bourget. Plusieurs explosions d'hydrogène eurent lieu dans les ateliers de Chalais-Meudon, dont l'une fit perdre un œil au capitaine Paul Renard.

13. «L'électricité dans ses premières grandeurs (1760-1820)», Revue d'histoire des sciences, t. 54, $\mathrm{n}^{\circ} 1,2001$.

14. Alain Beltran et Patrice Carré, La fée et la servante. La société française face à l'électricité (XIX-XX' siècle), Paris, Belin, 1991.

15. Maurice Lévy, "Conférence sur les unités électriques», Bulletin de la SEIN, 4 mars 1882, p. 233-259.

16. Gaston Tissandier, «Direction des aérostats par l'électricité», La Nature, n 418, 1881, p. 168.

17. Arthur Krebs, "Note sur un canot de 7 mètres avec machine électrique et accumulateurs", archives Krebs, pelurier (1886-1891), 17 avril 1888 ; cette note fait suite aux essais d'une chaloupe électrique de la marine servant de test préalable au sous-marin Gymnote.

18. Madeleine Akrich, Michel Callon, Bruno Latour, Sociologie de la traduction. Textes fondateurs, Paris, Mines, 2006, p. 271: «Dans ses études de l'innovation technologique, la Sociologie de l'Acteur Réseau met l'accent sur la capacité de chaque entité, spécialement les entités non humaines, à agir ou interagir d'une manière spécifique avec les autres humains ou non humains.» 
19. Wilfrid de Fonvielle, «L'aérostat dirigeable de Meudon", Le spectateur militaire, Paris, Ghio, 1884. Wilfrid de Fonvielle appartient au milieu des aéronautes privés qui, avec Giffard, Nadar, Godard et Tissandier, avant et pendant la guerre de 1870, ont porté les espoirs de la science aéronautique française, alors que l'administration restait sourde à leurs appels; journaliste scientifique, en 1884 , il possède une aura internationale.

20. M. Thébaud-Sorger, Une histoire des ballons..., op. cit., p. 146-147.

21. Fonds Renard: «[Krebs:] Il suffit de perfectionner ce qu'ont fait nos devanciers. Nous sommes certains d'obtenir un résultat»; Archives Krebs, Arthur Krebs, lettre autobiographique, 1924: «Pour donner confiance et obtenir des crédits, il fallait réussir et ne promettre que ce que l'on était sûr de pouvoir réaliser.»

22. Maryse Lassalle, Bases pour dirigeable, Histoire et actualités, Aix-en-Provence, Presses universitaires de Provence, 2005.

23. Larrie D. Ferreiro, "The mutual influence of aircraft aerodynamics and ship hydrodynamics in theory and experiment», Archive for the history of the exact sciences, $\mathrm{n}^{\circ} 68,2014$, p. 241-263.

24. Annales-industrielles, 7 septembre 1890, p. 290: «Une expérience du Gymnote. [...] Il revint à fleur d'eau à plus de 2 milles et demi de la passe par laquelle il était sorti et après être passé sous la ligne des torpilleurs sans avoir été vu. Une fois remonté à fleur d'eau pour reconnaître sa position, il se trouva avoir parcouru une ligne droite. Après avoir reconnu sa position, il plongea de nouveau pour se diriger du côté de la grande passe en traversant encore la ligne des torpilleurs. »

25. Archives du ministère de la Marine à Vincennes, 6W6, Lieutenant de vaisseau Darrieus [futur amiral], Étude sur les bateaux sous-marins, Paris, avril 1895, document héliogravé.

26. Konstantinos Chatzis, Georgia Mavrogonatou, «Eaux de Paris, eaux d'Athènes, 1830-1930: histoires croisées d'un réseau urbain", Almagest, International journal for the history of scientific ideas, 2010, n 1, p. 7-20.

27. D. Rodgers, Atlantic crossings. Social politics in a progressive age, Cambridge (Mass.), Harvard University Press, 1998.

28. Léon Donnat, La politique expérimentale, Paris, Reinwald (Bibliothèque des sciences con-

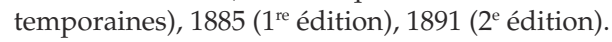

29. Christophe Studeny, L'invention de la vitesse, $\mathrm{XVIII}^{e}$-XXe siècle, Paris, Gallimard (NRF, Bibliothèque des histoires), 1995, p. 234: «Rapprochement magique des distances: les mœurs vont s'unifier par la circulation d'un fluide moral, les nations ne seront plus que des départements, les continents des provinces.»

30. Bulletin municipal officiel de la Ville de Paris, 28 juin 1887, p. 1555: «Ajournement d'une proposition de la $2^{\text {e }}$ Commission, relative au comité de perfectionnement des sapeurs-pompiers».

31. «Le matériel des pompiers de Paris, à propos de l'incendie de l'Opéra-Comique», $\mathrm{La}$ Nature, $\mathrm{n}^{\circ} 733,18$ juin 1887, p. 33.

32. Colonel Paris, Le feu à Paris et en Amérique, Paris, Germer Baillère et Cie, 1881.

33. Mathieu Flonneau, L'automobile à la conquête de Paris, 1910-1977. Formes urbaines, champ politique et représentations, 3 vol. dact. et illustrés, annexes documentaires et bibliographie, 1323 p., partiellement publié dans Mathieu Flonneau, L'automobile à la conquête de Paris. Chroniques illustrées, Paris, Presses de l'École nationale des Ponts et Chaussées, 2003, p. 102: "À vrai dire, l'implication des élus dans les affaires urbaines excédait de beaucoup le simple rôle délibératif qui leur était légalement dévolu par les préfectures. Leurs capacités de proposition étaient même très significatives dans le fonctionnement administratif, du fait des possibilités qu'ils se réservaient d'en appeler à la population en encadrant des pétitions.»

34. Archives de la Préfecture de police de Paris, DB273, Comité de perfectionnement du régiment de sapeurs-pompiers de Paris, Paris, Ville de Paris, 1888-1928, procès-verbal de la séance du 7 février 1889: «[Capitaine Krebs:] Les surfaces de ces périmètres seront proportionnées au nombre des habitants, ainsi qu'aux dangers d'incendie et de propagation du feu résultant du genre de construction». http://rytmo.net/ACK/1888-1928_ Pompiers_comite-perfectionnement.html.

35. Didier Rolland, La carrière militaire et industrielle d'Arthur Constantin Krebs (1870-1916), mémoire de maîtrise en histoire économique et sociale sous la dir. de Michel Lescure, université Paris-X Nanterre, 2002-2003, p. 75: «En treize années de service au Régiment, les fonctions clés qu'il a occupées lui ont permis de créer un service de lutte contre l'incendie original, dont il est prouvé aujourd'hui qu'il possédait la capacité de se développer. L'organisation actuelle s'appuie toujours sur les grands principes de la réorganisation Krebs». Didier Rolland est historien et major à la Brigade des sapeurs-pompiers de Paris.

36. Comité de perfectionnement..., op. cit., procèsverbal de la séance du 28 février 1889.

37. François Vatin, La fluidité industrielle. Essai sur la théorie de la production et le devenir du travail, Paris, Méridiens-Klincksieck, 1987. 
38. Matthieu Flonneau, Vincent Guigueno (éd.), De l'histoire des transports à l'histoire de la mobilitée, Rennes, Presses universitaires de Rennes, 2009.

39. Mathieu Flonneau, «Paris au cœur de la révolution des usages de l'automobile, 18841908», Histoire, économie et société, 2007, n² 2, p. 61-74.

40. Brevet français $\mathrm{n}^{\circ} 256.344$ du 13 mai 1896: Système de voiture automobile; ce brevet a également été déposé au Royaume-Uni par Krebs et en Belgique par Levassor.

41. U. S. Circuit Court, Southern district of New York, Equity case 8616, op. cit., p. 2: «Thereafter I occupied myself trying to propel vehicles by means of motors. It is thus that about 1884 I followed with great interest the construction of the small petrol motors of Monsieur Daimler. In 1889 in the shop of Panhard-Levassor was made the first application of this Daimler engine to a vehicle. This firm had also made gas engines of the Otto type and Benz type which they abandoned to give their entire attention to the Daimler motor. »

42. Archives Krebs; ce dessin intitulé «Projet de voiture automobile», daté de 1868, peut avoir été exécuté dans le cadre des études de Math-Spé de Krebs à Besançon.

43. U. S. Circuit Court, Southern district of New York, Equity case 8616, op. cit., p. 69: «A127. The engine which I studied at that time was not a 4-cycle engine, but an engine with combustion at constant pressure. [...] A128. I said that in 1878 I purchased an Otto shop engine for use in our balloon factory, while studying later on light engines I had neither the experience nor above all the genius of Daimler. I was evidently following on the wrong track, and succeeded ultimately in the object I had in view (steering balloons), by using an electric motor.»

44. Le Génie français et l'Ingénieur civil réunis,

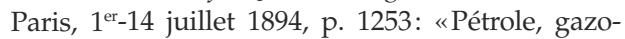
line, essence de pétrole, pétrole ou gaz, système Serpollet et pétrole combinés, gaz et pesanteur, air comprimé, vapeur, air comprimé ou gaz, un gaz sous forte pression, roue à vapeur, vapeurs combinées, moteur à liquides combinés, moteurs électriques, moteur semi-électrique, moteurs hydrauliques, eau comprimée, combinaison d'un moteur animé et d'un moteur mécanique, la pesanteur, le poids des voyageurs, systèmes de leviers, systèmes de balanciers, système de pédale, moteur à propulsion constante, voiture fournissant ellemême son moteur, moteurs automatiques.»

45. Pierre Souvestre, Histoire de l'automobile, Paris, Dunot et Pinat, 1907, p. 280.
46. Le Français Charles Terront gagne la course cycliste Paris-Brest de 1891 (1200 km en 71h30 $\mathrm{mn}$ ) à la moyenne de $17 \mathrm{~km} / \mathrm{h}$. La même année, le quadricycle Peugeot à moteur Daimler-Panhard parcourt le trajet Valentigney-Brest et retour à la vitesse moyenne de $15 \mathrm{~km} / \mathrm{h}$. Le puissant attelage de Dion à vapeur, de même que les voitures Peugeot et Panhard à moteurs à pétrole Daimler, effectuent en 1894 les 126 km de Paris à Rouen avec une moyenne d'environ $22 \mathrm{~km} / \mathrm{h}$.

47. La Nature, $\mathrm{n}^{\circ} 1104,28$ juillet 1894, p. 130: "Quels sont, en effet, les systèmes moteurs - en petit nombre - auxquels on peut avoir recours pour mouvoir un véhicule autonome? ».

48. U. S. Circuit Court, Southern district of New York, Equity case 8616, op. cit., p. 68: «A122. I have known Mr. Daimler in 1897, when I went to visit him at Cannstadt, Germany, in October. In the house he lived in, he showed me the laboratory and shop where he had studied and first built his little engine, then the first vehicle on which he made his first trials.» À noter que Gottlieb Daimler est actionnaire et administrateur de la nouvelle société anonyme Panhard-Levassor.

49. Comité de perfectionnement, op. cit., procèsverbal de la séance du 5 juillet 1895: «L'industrie ne construit actuellement que des moteurs de 2 à 3 chevaux. Or, c'est une force de 12 à 15 chevaux qu'il faut au Corps.»

50. Archives de la Préfecture de police de Paris, DB270, article de journal du 16 mars 1902 intitulé «L'attaque rapide du feu».

51. Henry Le Chatelier, "Du rôle de la science dans l'industrie", Revue de métallurgie, vol. $1, \mathrm{n}^{\circ} 1$, 1904, p. 1-10.

52. Arthur Krebs, «Sur un carburateur automatique pour moteurs à explosions. Note de M. Krebs, présentée par M. Maurice Lévy", Comptes rendus des séances de l'Académie des sciences, Paris, Gauthier-Villars, 24 novembre 1902; ce carburateur initie la classe des carburateurs à dépression constante.

53. Brevet français $n^{\circ} 301.991$ du 7 juillet 1900 : Système de régulateur électro-magnétique agissant sur les soupapes d'admission, pour moteurs à hydrocarbures. Krebs dépose également un brevet $n^{\circ} 439.445$ du 6 avril 1911: Dispositif de prise directe applicable aux appareils de changement de vitesse par fluide.

54. Citons le brevet $\mathrm{n}^{\circ} 316.326 \mathrm{du} 16$ novembre: Appareil de distribution du courant primaire pour allumage par bobine et bougie dans les moteurs à explosion.

55. Nous pouvons nous représenter cet impact de l'allumage électrique sur le moteur à pétrole 
comme analogue à l'apparition de l'allumage électronique sur les moteurs à essence dans les années 1980.

56. Gérard Lavergne, Manuel théorique et pratique de l'automobile sur route, Paris, Librairie polytechnique, 1900, p. 19: «[...] il ne faut pas compter actuellement sur plus de $11 \%$ sur l'arbre du moteur [à pétrole] et 5,5\% seulement aux jantes, à cause des pertes occasionnées par la transmission.»; ibid., p. 204: «[...] le rendement final [du gros moteur Diesel] n'en atteint pas moins 0,266, ce qui veut dire que $26,6 \%$ de la chaleur du combustible est bel et bien transformée en travail effectif au frein».

57. Marcel Déprez, «Nouvelles expressions $\mathrm{du}$ travail et du rendement économique du travail électrique», La lumière électrique, 1882, p. 564. En 1882, Marcel Déprez réalise un "frein dynamo-électrique» de laboratoire dans le cadre de ses travaux sur la récente adoption, en 1881, des unités internationales de mesure en électricité.

58. Arthur Krebs, "Sur un frein dynamométrique destiné à la mesure de la puissance des moteurs, qui permet l'utilisation, sous forme électrique, de la majeure partie du travail développé. Note de M. A. Krebs présentée par M. d'Arsonval», Comptes rendus des séances de l'Académie des sciences, Paris, Gauthier-Villars, 13 novembre 1905. Ce type de frein est aujourd'hui connu sous les noms de "dynamo balance», "génératrice balance», «génératrice dynamométrique», «dynamo-frein», «frein électromagnétique» ou même «électrodynamomètre».

59. L'électricité produite lors de cette mesure est injectée dans le réseau de l'usine qui produit par ailleurs sa propre électricité grâce à de puissantes machines à vapeur.

60. The horseless age, New York, 2 juillet 1902: «Panhard \& Levassor are said to have acquired the Lohner-Porsche patents on a combination electric and gasoline system for France, England and Italy». «A 15-H.P. Lohner-Porsche petrol-electric car», The Automotor Journal, 4 avril 1903, p. 352. Noter que ce véhicule est équipé du nouveau carburateur automatique.

61. Gilbert Simondon. Du mode d'existence des objets techniques, Paris, Aubin, 1989; L'invention dans les techniques, Paris, Seuil, 2005.

62. J. Mathiot, «Humeurs fluides...», op. cit., p. 60.

63. Zygmunt Bauman, Liquid modernity, Cambridge, Polity Press, 2000.

64. Parmi les membres de la Section des recherches psychiques et physiologiques de l'Institut général psychologique, on compte les personnalités suivantes: les physiciens Branly et Perrin, le philosophe Henri Bergson, ainsi que le philosophe des sciences Georges Matisse.

65. Jules Courtier, Rapport sur les séances d'Eusapia Palladino à l'Institut général psychologique en 1905, 1906, 1907 et 1908, Institut général psychologique, Section des recherches psychiques et physiologiques, Paris, 1909, p. 415 et 476.

66. Albert Einstein, "Zur Elektrodynamik bewegter Körper», Annalen der Physik, vol. 17, 30 juin 1905, p. 891-921. Extrait de l'article espacetemps, Wikipedia.fr, 11/2013: «En physique, l'espace-temps est une représentation mathématique de l'espace et du temps comme deux notions inséparables et s'influençant l'une l'autre. [...] Le continuum espace-temps comporte quatre dimensions: trois dimensions pour l'espace, « $\mathrm{x} »,\langle\mathrm{y} »$, et « $\mathrm{Z}$ », et une pour le temps, « $\mathrm{t} »$. Afin de pouvoir les manipuler plus aisément, on s'arrange pour que ces quatre grandeurs soient homogènes à une distance en multipliant « $\mathrm{t}$ » par la constante "C 》 (vitesse de la lumière dans le vide).»

67. Philippe Krebs, Arthur Constantin Krebs (1850-1935). Autorité et stratégie à la direction de Panhard et Levassor (1913-1916), mémoire de master II, sous la dir. de Liliane Pérez, CDHTE, Conservatoire national des arts et métiers, Paris, 2009 <http://www.sudoc.fr/159848989>. 